\title{
Original
}

\section{Effect of Repeated Beta-Phenylethylamine or Methamphetamine Administration on Brain Monoamine Oxidase Activity}

\author{
Mitsuhiko Yamada, Yuji Kivchi, Miyuki Hashimoto, Yumi KamiJo, \\ Daichi Morioka, Hajime Yasuhara and Katsuji OguchI
}

\begin{abstract}
We previously demonstrated that changes in brain monoamine levels 4 weeks after withdrawal from repeated administration of beta-phenylethylamine (PEA) are different from changes 4 weeks after methamphetamine (MAP) withdrawal. Monoamine oxidase (MAO) is physiologically important in the metabolism of monoamines. In the present study, we measured MAO activity radiometrically 4 weeks after the withdrawal from repeated PEA or MAP administration in rats. Reverse tolerance to stereotyped behavior was induced after repeated administration of PEA or MAP in rats and was maintained for at least 4 weeks after withdrawal of either drug. To measure MAO activity, either 5-hydroxytryptamine (5-HT) (a specific substrate for type A MAO), or benzylamine (a specific substrate for type B MAO) was used as a substrate. There was no significant change in MAO activity after withdrawal of either drug. We concluded that MAO is not responsible for the changes in brain monoamine levels observed after withdrawal from repeated PEA or MAP administration in rats.
\end{abstract}

Key words: beta-phenylethylamine, methamphetamine, reverse tolerance, monoamine oxidase, behavior

\section{Introduction}

Beta-phenylethylamine (PEA) is a trace amine which is naturally present in the mammalian brain $^{1)}$. Although PEA structurally resembles amphetamine (AMPH), and possesses similar pharmacological properties, the physiological roles of PEA are not as fully understood.

After repeated administration of PEA in rats, augmented stereotyped behavior is observed $^{2,3)}$. The same phenomenon is observed after repeated administration of AMPH or methamphetamine (MAP) ${ }^{4-i)}$, and is called "reverse tolerance". It is proposed that the reverse tolerance to stereotyped behavior in animals may closely resemble AMPH psychosis in man, because of the similarity between the behavioral sensitization and relapse in psychosis $^{8,9)}$.

We previously demonstrated that reverse tolerance to stereotyped behavior is induced after repeated administration of $\mathrm{PEA}^{3)}$ or $\mathrm{MAP}^{4)}$ in rats, and the changes in brain monoamine levels during withdrawal are different for each drug. Monoamine oxidase (MAO) is physiologically important in the metabolism of monoamines. In the present study, we measured MAO activity 4 weeks after withdrawal from repeated administration of either PEA or 
MAP in rats.

\section{Materials and Methods}

Animals. Male Wistar, 7-week-old rats, weighing 200-250 g at the beginning of the study were used. They had continuous access to food and water except during the experimental session, and were on a 12-hour light/dark cycle under standard laboratory conditions.

Groups and schedule. The rats were divided into 2 groups. In one group $(n=6)$, reverse tolerance to stereotyped behavior was induced by repeated administration of PEA. MAO activity was measured 4 weeks after PEA withdrawal. In the other group $(n=6)$, the process was repeated with MAP.

Induction of reverse tolerance to stereotyped behavior. In the PEA group, $50 \mathrm{mg} / \mathrm{kg}$ PEA, or $1 \mathrm{ml} / \mathrm{kg}$ saline as a control, was administrated i.p., daily for ten days. To confirm the induction of reverse tolerance, the changes in stereotyped behavior were rated after each administration on the first, second, third, fifth and the tenth days using a five-point stereotypy scale $^{3)}$ modified from the method of Borison et al. ${ }^{10)}$. The same dose $(50 \mathrm{mg} / \mathrm{kg}$ i.p.) was rechallenged 4 weeks after the withdrawal, and streotyped behavior was scored again to confirm maintenance of the reverse tolerance.

In the MAP group, rats were administrated MAP or $1 \mathrm{ml} / \mathrm{kg}$ saline as a control, every other day. On the first day, $2.5 \mathrm{mg} / \mathrm{kg}$ MAP was administered i.p. 3 times a day. The doses were increased to $5 \mathrm{mg} / \mathrm{kg}$ on the third day and to $7.5 \mathrm{mg} / \mathrm{kg}$ on the fifth day. On the seventh day, $10 \mathrm{mg} / \mathrm{kg}$ MAP was administered i.p., two times. To confirm the induction of reverse tolerance, $2 \mathrm{mg} / \mathrm{kg}$ MAP was rechallenged i.p. on the eighth day and 4 weeks after the withdrawal, and stereotyped behavior was scored using a ten-point streotypy scale according to Ellinwood and Balster ${ }^{6}$.

At least 2 hours prior to the observation, the rats were placed in individual experimental cages and allowed to adapt to the observation room.

Enzyme preparation. The rats were sacrificed by decapitation; the whole brain was removed immediately, placed on an ice plate, and dissected into seven parts ${ }^{11}$. The cerebral cortex, midbrain with thalamus, hypothalamus and striatum were used for the enzyme materials. The tissues were weighed and homogenates were prepared in 10 volumes of $0.01 \mathrm{M}$ phosphate buffer ( $\mathrm{pH}$ 7.4) using a glass homogenizer.

Enzyme assay. MAO activity was determined radiometrically by the method of Wurtman and Axelrod ${ }^{12)}$ with $\left[{ }^{14} \mathrm{C}\right]$ labeled 5-hydroxytryptamine (5-HT) (type A MAO) or benzylamine (type B MAO) as substrates. Incubation was carried out at $37^{\circ} \mathrm{C}, \mathrm{pH} 7.4$ for $10 \mathrm{~min}$. In this assay, enzyme activity is linearly proportional to the amount of protein and to time, throughout all experiments. Protein concentration was determined using the modified method of Lowry et al.:i:) with bovine serum albumin as a standard. Statistical evaluations were determined by Student's $t$-test.

Drugs. The following drugs were used: $\left[{ }^{14} \mathrm{C}\right]-5-\mathrm{HT}$ binoxalate was purchased from New England Nuclear (Boston, MA) and $\left[{ }^{14} \mathrm{C}\right]$-benzylamine hydrochloride was purchased from Amersham International, Ltd. (Buckinghamshire, UK). Other chemicals used were of the highest grade commercially available.

\section{Results}

Induction of reverse tolerance. As shown in Fig. 1, the mean maximum score increased 


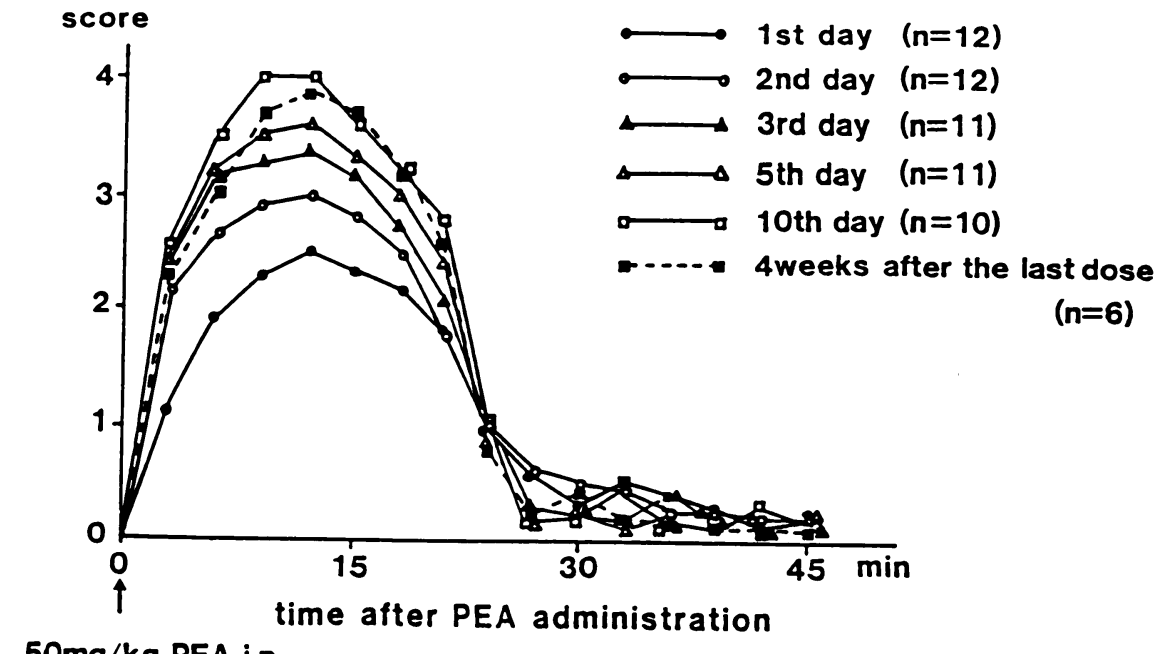

\section{$50 \mathrm{mg} / \mathrm{kg}$ PEA i.p.}

Fig. 1. Stereotyped behavior after each PEA administration.

Changes in the stereotyped behavior were scored every $3 \mathrm{~min}$ for $45 \mathrm{~min}$ after each PEA administration ( $50 \mathrm{mg} / \mathrm{kg}$ i.p.) on the first, second, third, fifth, tenth day and 4 weeks after the withdrawal using five-point stereotypy scale ${ }^{3)}$. It is confirmed that reverse tolerance to stereotyped behavior after repeated PEA administration was maintained at least for 4 weeks.

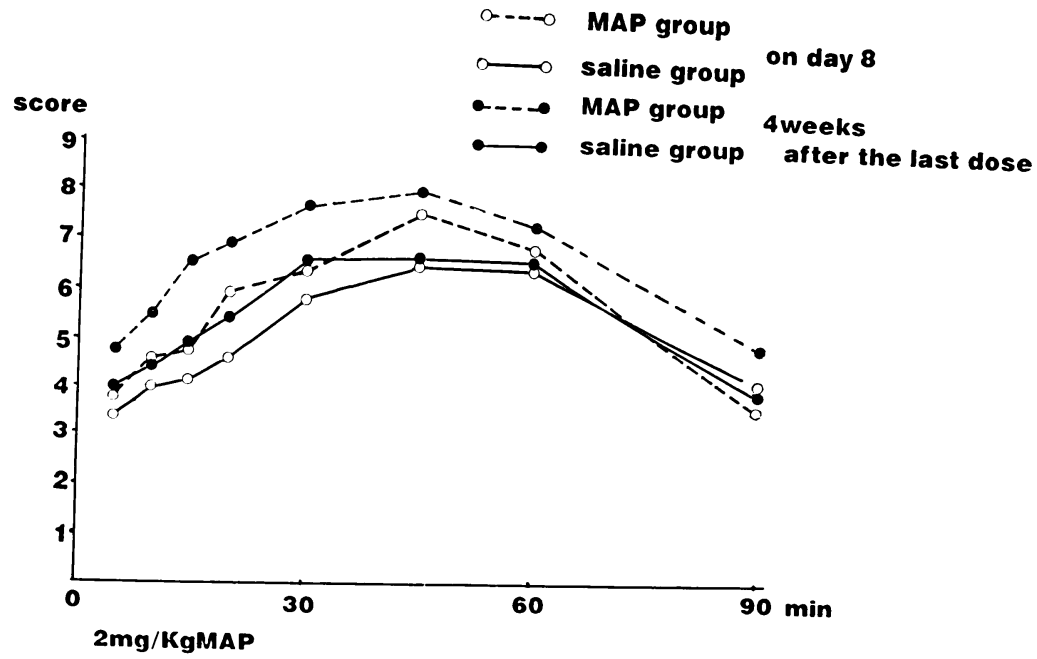

Fig. 2. Stereotyped behavior after each MAP administration.

Changes in the stereotyped behavior were scored for $120 \mathrm{~min}$ after each MAP administration ( $2 \mathrm{mg} / \mathrm{kg}$ i.p.) on 2 days and 4 weeks after the withdrawal using ten-point stereotypy scale according to Ellinwood and Balster ${ }^{(i)}$. It is confirmed that reverse tolerance to stereotyped behavior after repeated MAP administration was maintained at least for 4 weeks. 
Table 1. Monoamine oxidase activity in the brain 4 weeks after PEA withdrawal in rats.

\begin{tabular}{lccccc} 
& \multicolumn{2}{c}{$5-\mathrm{HT}$} & & Benzylamine \\
\cline { 2 - 3 } \cline { 5 - 6 } & Control $(\mathrm{n}=6)$ & PEA $(\mathrm{n}=4)$ & & Control $(\mathrm{n}=6)$ & PEA $(\mathrm{n}=4)$ \\
Midbrain with thalamus & $0.25 \pm 0.04$ & $0.27 \pm 0.05$ & & $0.34 \pm 0.14$ & $0.40 \pm 0.05$ \\
Hypothalamus & $0.23 \pm 0.05$ & $0.23 \pm 0.07$ & & $0.32 \pm 0.11$ & $0.35 \pm 0.02$ \\
Striatum & $0.34 \pm 0.04$ & $0.36 \pm 0.08$ & & $0.54 \pm 0.04$ & $0.57 \pm 0.06$ \\
& $0.25 \pm 0.03$ & $0.29 \pm 0.05$ & & $0.34 \pm 0.02$ & $0.39 \pm 0.05$
\end{tabular}

Rats were decapitated 4 weeks after PEA withdrawal and monoamine oxidase activity was measured by radiometric assay using 5-HT or benzylamine as substrate. Results are shown as mean \pm S.E.M. $(\mathrm{nmol} / \mathrm{mg}$ protein $/ \mathrm{min})$. No statistical difference was found (Student's t-test).

Table 2. Monoamine oxidase activity in the brain 4 weeks after MAP withdrawal in rats.

\begin{tabular}{|c|c|c|c|c|}
\hline & \multicolumn{2}{|c|}{ 5-HT } & \multicolumn{2}{|c|}{ Benzylamine } \\
\hline & Control $(n=6)$ & MAP $(n=6)$ & Control $(n=6)$ & MAP $(n=6)$ \\
\hline Cerebral cortex & $0.26 \pm 0.01$ & $0.24 \pm 0.01$ & $0.39 \pm 0.04$ & $0.38 \pm 0.04$ \\
\hline Midbrain with thalamus & $0.24 \pm 0.03$ & $0.22 \pm 0.02$ & $0.34 \pm 0.07$ & $0.33 \pm 0.04$ \\
\hline Hypothalamus & $0.28 \pm 0.03$ & $0.25 \pm 0.07$ & $0.44 \pm 0.06$ & $0.44 \pm 0.07$ \\
\hline Striatum & $0.25 \pm 0.03$ & $0.21 \pm 0.02$ & $0.36 \pm 0.02$ & $0.34 \pm 0.03$ \\
\hline
\end{tabular}

Rats were decapitated 4 weeks after MAP withdrawal and monoamine oxidase activity was measured by radiometric assay using 5-HT or benzylamine as substrate. Results are shown as mean \pm S.E.M. (nmol/mg protein/min). No statistical difference was found (Student's t-test).

progressively with time. The time to reach the maximum score, and the duration of the response did not change throughout the experiment. Four weeks after withdrawal, augmented stereotyped behavior was again observed after $50 \mathrm{mg} / \mathrm{kg}$ injection of PEA, the same dose as before. The mean maximum score was still high, showing that reverse tolerance to stereotyped behavior after PEA withdrawal was maintained for at least 4 weeks.

As shown in Fig. 2, 2 days after the MAP withdrawal, the mean maximum score was higher than that of the saline treated group after $2 \mathrm{mg} / \mathrm{kg}$ injection of MAP, i.p. Four weeks after withdrawal, augmented stereotyped behavior was also observed after injection of $2 \mathrm{mg} / \mathrm{kg}$ MAP, the same dose as before, showing that reverse tolerance to stereotyped behavior after MAP withdrawal was maintained for at least 4 weeks.

$M A O$ activity. As shown in Table 1, 4 weeks after PEA withdrawal, MAO specific activity using both 5-HT and benzylamine as substrates had not changed significantly. As shown in Table 2, MAO specific activity using both 5-HT and benzylamine as substrates had not changed significantly 4 weeks after MAP withdrawal.

\section{Discussion}

In this study, we demonstrated that reverse tolerance to stereotyped behavior was induced after repeated PEA or MAP administration in rats and it persisted for at least four weeks. This is consistent with previous reports ${ }^{2-5)}$. The augmented behavioral response after repeated PEA or MAP treatment can be explained by enhanced dopamine (DA) release as observed after repeated AMPH $^{5,8,14)}$.

We previously reported that changes in brain monoamine levels at the withdrawal period 
were different after each drug administration. A significant increase of noradrenaline (NA) in the cerebral cortex and a decrease of 5-HT in the hypothalamus were found 4 weeks after PEA withdrawal. Four weeks after MAP withdrawal, significant decreases in NA in the striatum, 5-HT in the cerebral cortex and striatum and 5-hydroxyindoleacetic acid in the cerebral cortex and midbrain with thalamus were found. It is interesting that the levels of dopamine, 3,4-dihydroxyphenylacetic acid and homovanillic acid did not change in any brain region long after withdrawal from $\mathrm{PEA}^{:)}$or $\mathrm{MAP}^{4)}$ treatment, though some neurochemical changes are proposed in dopaminergic nerve terminals.

In the present study, we measured MAO activity in rats 4 weeks after withdrawal from PEA or MAP. MAP is known to be a potent inhibitor of $\mathrm{MAO}^{15)}$, but there was no significant change in specific MAO activity using either 5-HT (type A MAO) or benzylamine (type B MAO) as a substrate after MAP withdrawal. There was also no significant change in specific MAO activity using either 5-HT or benzylamine as a substrate after PEA withdrawal. PEA is known to be a good substrate for type $\mathrm{B} \mathrm{MAO}^{16)}$; it seems that rechallenged PEA is not affected by the altered activity of type B MAO. It is proposed that the changes in brain monoamine level after withdrawal from either drug would not be related to MAO.

Previously, we also demonstrated that a lack of hyporesponsiveness to acoustic stimulation, which had been observed after withdrawal from repeated MAP treatment ${ }^{4)}$, was evident after withdrawal from PEA"). It is also suggested that MAO is irrelevant to the mechanisms that contribute to this behavioral difference.

In conclusion, it is suggested that MAO is not responsible for the changes in brain monoamine levels observed after withdrawal from repeated PEA or MAO administration in rats.

\section{References}

1) Nakajima T, Kamimoto $Y$ and Sano I: Formation of $\beta$-phenylethylamine in mammalian tissue and its effect on motor activity in the mouse. J Pharmacol Exp Ther, 143: 319-325 (1964)

2) Kuroki T, Tsutsumi T, Hirano M, Matsumoto T, Tatebayashi Y, Nishiyama K, Uchimura H, Shiraishi A, Nakahara $T$ and Nakamura $K$ : Behavioral sensitization to beta-phenylethylamine (PEA): enduring modifications of specific dopaminergic neuorn systems in the rat. Psychopharamacol, 102: 5-10 (1990)

3) Yamada M, Kiuchi Y, Hashimoto M, Oguchi $\mathrm{K}$ and Yasuhara H: The response to acoustic stimulation and the changes in brain amine levels after repeated administration of $\beta$-phenylethylamine in rats. Jpn J Pharmacol, 56: 127-132 (1991)

4) Akita H, Hashimoto M, Yamada M, Kiuchi Y, Oguchi K and Yasuhara H: Behavioral characteristics associated with acoustic stimulation and the neurochemical alterations of monoaminergic systems in rat brain at the steady state of repeated methamphetamine administration. Folia Pharmacol Jpn, 95: 327-333 (1990) (in Japanese)

5) Ichikawa $\mathbf{J}$ : Change of behavior and central monoaminergic systems in the rat after repeated methamphetamine pretreatment: Presynaptic regulatory mechanism. Jpn J Psychopharmacol, 8: 389403 (1988)

6) Ellinwood EHJr and Balster RL: Rating the behavioral effects of amphetamine. Eur J Pharmacol, 28: 35-41 (1974)

7) Robinson TE and Becker JB: Enduring changes in brain and behavior produced by chronic amphetamine administration: a review and evaluation of animal models of amphetamine psychosis. Brain Res Rev, 11: 157-198 (1986)

8) Kokkinidis L and Anisman H: Amphetamine models of paranoid schizophrenia: an overview and elaboration of animal experiment. Psychol Bull, 88: 551-579 (1980)

9) Bell DS: The experimental reproduction of amphetamine psychosis. Arch Gen Psychiatr, 29: 35-40 (1973)

10) Borison RL, Havdala HS and Diamond BI: Chronic phenylethylamine stereotypy in rats: A new 
animal model for schizophrenia? Life Sci, 21: 117-122 (1977)

11) Glowinski J and Iversen LL: Regional studies of catecholamines in the rat brain-I; The disposition of $\left[{ }^{3} \mathrm{H}\right]$ norepinephrine, $\left[{ }^{3} \mathrm{H}\right]$ dopamine and $\left[{ }^{3} \mathrm{H}\right]$ dopa in various region of the brain. J. Neurochem, 13: $655-669$ (1966)

12) Wurtman RJ and Axelrod J: A sensitive and specific assay for the estimation of monoamine oxidase. Biochem Pharmacol, 12: 1439-1441 (1963)

13) Lowry OH, Rosebrough NT, Farr AL and Randall RJ: Protein measurement with the folin phenol reagent. J Biol Chem, 193: 265-275 (1951)

14) Nishikawa T, Mataga N, Takashima $M$ and Toru $M$ : Behavioral sensitization and relative hyperresponsiveness of striatal and limbic dopaminergic neurons after repeated methamphetamine treatment. Eur J Pharmacol, 88: 195-203 (1983)

15) Utena $\mathbf{H}$, Ezoe $T$, Kato $\mathbf{N}$ and Hada $\mathbf{H}$ : Effects of administration of methamphetamine in enzymic patterns in brain tissue. J Neurochem, 4: 161-169 (1959)

16) Yang HYT and Neff NH: Beta-phenylethylamine, a specific substrate for type B monoamine oxidase of brain. J Pharmacol Exp Ther, 187: 365-371 (1973)

[Received May 21, 1991: Accepted June 22, 1991] 